\title{
CONJUGACY CLASS SIZE CONDITIONS WHICH IMPLY SOLVABILITY
}

\author{
QINGJUN KONG ${ }^{\otimes}$ and QINGFENG LIU
}

\begin{abstract}
(Received 26 August 2012; accepted 13 September 2012; first published online 15 January 2013)
The paper is dedicated to Professor John Cossey for his 70th birthday
\end{abstract}

\begin{abstract}
Let $G$ be a finite $p$-solvable group and let $G^{*}$ be the set of elements of primary and biprimary orders of $G$. Suppose that the conjugacy class sizes of $G^{*}$ are $\left\{1, p^{a}, n, p^{a} n\right\}$, where the prime $p$ divides the positive integer $n$ and $p^{a}$ does not divide $n$. Then $G$ is, up to central factors, a $\{p, q\}$-group with $p$ and $q$ two distinct primes. In particular, $G$ is solvable.
\end{abstract}

2010 Mathematics subject classification: primary 20E45; secondary 20D10.

Keywords and phrases: conjugacy class sizes, solvable groups, finite groups.

\section{Introduction}

Throughout the following, $G$ always denotes a finite group and $p$ a prime. For an element $x$ of a group $G$, the conjugacy class of $x$ is the set $x^{G}=\left\{x^{g} \mid g \in G\right\}$ and the size of this class is called the index of $x$ in $G$ [2]. We say that a group element has primary, biprimary or triprimary order if its order is divisible by at most one, two or three primes, respectively. The rest of our notation and terminology are standard.

A classic problem in finite group theory is to study the influence of the conjugacy class sizes on the structure of a group. For example, groups with two class sizes are nilpotent [6] and groups with three class sizes are solvable [7]. But in general, groups with four class sizes need not be solvable and can even be simple. In 1970, Itô in [8] showed that the groups $\operatorname{SL}\left(2,2^{m}\right)$ for $m \geq 2$ are the only simple groups with four class sizes. On the other hand, Beltrán and Felipe prove in [3] that when the conjugacy class sizes of $G$ are precisely $\left\{1, p^{a}, n, p^{a} n\right\}$ with $(p, n)=1$ and $a \geq 0$, then $G$ is solvable. Furthermore in the same paper, they prove that when the conjugacy class sizes of $G$ are precisely $\{1, m, n, m n\}$ with $(m, n)=1$, then $G$ is also solvable. In $[9,10]$ the first author extends the former result by replacing conditions for all conjugacy classes by conditions referring to only some conjugacy classes. For instance, the conjugacy

The research of the authors is supported by the National Natural Science Foundation of China (10771132), SGRC (GZ310), the Research Grant of Tianjin Polytechnic University.

(C) 2012 Australian Mathematical Publishing Association Inc. 0004-9727/2012 \$16.00 
classes of elements of primary, biprimary and triprimary orders of $G$. The main results are the following theorems.

Theorem A [9, Theorem A]. Let $G$ be a group and let $G^{*}$ be the set of elements of primary, biprimary and triprimary orders of $G$. Suppose that the conjugacy class sizes of $G^{*}$ are precisely $\left\{1, p^{a}, n, p^{a} n\right\}$ with $(p, n)=1$ and $a \geq 0$. Then $G$ is solvable.

Theorem B [10, Theorem A]. Let $G$ be a group and let $G^{*}$ be the set of elements of primary, biprimary and triprimary orders of $G$. Suppose that the conjugacy class sizes of elements of $G^{*}$ are precisely $\left\{1, p^{a}, n, p^{a} n\right\}$ with $(p, n)=1$ and $a \geq 0$. Then $G$ is nilpotent and $n=q^{b}$ for some prime $q$.

An obvious question about solvability arises when we eliminate the coprime hypothesis, and we are interested in studying which arithmetical conditions on groups with four class sizes of elements of primary, biprimary and triprimary orders of $G$ yield their solvability. In this paper, we eliminate the coprime hypothesis $(p, n)=1$ and class sizes of elements of triprimary orders of $G$. Our main result is the following theorem.

Theorem C. Let $G$ be a p-solvable group and let $G^{*}$ be the set of elements of primary and biprimary orders of $G$. Suppose that the conjugacy class sizes of $G^{*}$ are $\left\{1, p^{a}, n, p^{a} n\right\}$, where $p$ divides the positive integer $n$ and $p^{a}$ does not divide $n$. Then $G$ is, up to central factors, $a\{p, q\}$-group with $p$ and $q$ two distinct primes. In particular, $G$ is solvable.

We believe that the result is true for arbitrary integers $p$ and $n$, but we have not been able to prove it with the techniques we employ here.

\section{Basic definitions and preliminary results}

In this section, we state the necessary results for the proof of our main theorem.

The following result determines some properties of the structure of groups having precisely two conjugacy class sizes of $p^{\prime}$-elements of primary orders for some prime $p$.

Lemma 2.1 [1, Theorem A]. Suppose that $G$ is a finite p-solvable group and that 1 and $m$ are the conjugacy class sizes of $p^{\prime}$-elements of prime power order. Then $m=p^{a} q^{b}$, with $q$ a prime distinct from $p$, and $a, b \geq 0$. If $b=0$, then $G$ has abelian $p$-complement. If $b \neq 0$, then $G=P Q \times A$, with $P$ and $Q$ a Sylow $p$-subgroup and a Sylow $q$-subgroup of $G$, respectively, and $A \leq Z(G)$. Furthermore, if $a=0$, then $G=P \times Q \times A$.

Lemma 2.2 [11, Lemma 2.4]. Let $G$ be a group. A prime $p$ does not divide any conjugacy class length of any element of prime power order of $G$ if and only if $G$ has a central Sylow p-subgroup.

Lemma 2.3 [5, Lemma 1]. Let $K \unlhd G$, where $G$ is an arbitrary finite group and $K$ is abelian. Let $x$ be a noncentral element of $G$, and let $y=[t, x]$ for some element $t \in K$. Then $\left|C_{G}(y)\right|>\left|C_{G}(x)\right|$, and so the $G$-class of $y$ is smaller than that of $x$. 
Lemma 2.4 [4, Lemma 5]. Let $x$ and $y$ be a p-element and a p'-element, respectively, of a group $G$, such that $[x, y]=1$ and $C_{G}(x) \subseteq C_{G}(y)$. Then $O_{p}(G) \subseteq C_{G}(y)$.

\section{Proof of Theorem C}

By the condition of Theorem $\mathrm{C}$, we assume that $p$ divides the positive integer $n$, but that $p^{a}$ does not divide $n$. We need to distinguish two cases to complete the proof of the main theorem.

Case 1. First we assume that there are no $p$-elements of index $p^{a}$. Notice that the centraliser of any element of index $p^{a}$ is a maximal subgroup among all centralisers, so by considering the primary decomposition we can assume without loss of generality that $x$ is a $q$-element for some prime $q \neq p$ such that $\left|x^{G}\right|=p^{a}$. Let $y$ be a $q^{\prime}$-element of primary order of $C_{G}(x)$. Then

$$
C_{G}(x y)=C_{G}(x) \cap C_{G}(y) \subseteq C_{G}(x)
$$

and, since $p^{a}$ does not divide $n$, the index of $y$ in $C_{G}(x)$ must be 1 or $n$. If there exists any $q^{\prime}$-element of primary order of $C_{G}(x)$ of index $n$, then $n$ is the product of at most two prime powers by Lemma 2.1, say $n=q^{b} r^{c}$. In this case, necessarily $r=p$, and then $G$ is a $\{p, q\}$-group, up to central factors, by Lemma 2.2 and the proof is complete. If there is no $q^{\prime}$-element of primary order in $C_{G}(x)$ of index $n$, then we can write $C_{G}(x)=Q_{x} \times H_{x}$, with $H_{x}$ an abelian $q^{\prime}$-complement of $C_{G}(x)$. As $p$ divides $n$, it follows that $p^{a}<p^{a} n_{p} \leq|G: Z(G)|_{p}$ and this yields the fact that $p$ divides $\left|C_{G}(x) / Z(G)\right|$. Hence, there exists a $p$-element in $C_{G}(x)$, say $t$, such that $C_{G}(x)=C_{G}(t)$, so $t$ is a $p$ element of index $p^{a}$, which is impossible.

Case 2. Now suppose that there is a $p$-element $z$ such that $\left|z^{G}\right|=p^{a}$. Then, if $y$ is a $p^{\prime}$-element of primary order of $C_{G}(z)$, we get

$$
C_{G}(z y)=C_{G}(z) \cap C_{G}(y) \subseteq C_{G}(z) .
$$

As in the previous paragraph, $y$ has index 1 or $n$ in $C_{G}(z)$, and if there are $p^{\prime}$ elements of primary orders of both indexes 1 and $n$ in $C_{G}(z)$, it follows that $n=p^{a} q^{b}$ by Lemma 2.1. By Lemma 2.2, we conclude that $G$ is a $\{p, q\}$-group (or a $p$ group if $b=0$ ), up to central factors. Suppose now that every $p^{\prime}$-element of primary order of $C_{G}(z)$ has index 1. Accordingly, we can write $C_{G}(z)=P_{z} \times H_{z}$, where $H_{z}$ is an abelian $p$-complement. As $C_{G}(z)$ has index $p^{a}$ in $G$, we deduce that $G$ has abelian $p$-complements, so in particular $G$ is solvable as it is the product of two nilpotent subgroups. Now let us consider $O_{p^{\prime}}(G)$. Since $O_{p^{\prime}}(G)$ is an abelian normal subgroup of $G$, by applying Lemma 2.3 we can obtain $\left|C_{G}([w, t])\right|>\left|C_{G}(w)\right|$ for every $t \in G$ and for every $w \in O_{p^{\prime}}(G)$ that is noncentral in $G$. Then the hypotheses of the theorem imply that $\left[O_{p^{\prime}}(G), G\right] \subseteq Z(G)$. Moreover, by coprime action, $O_{p^{\prime}}(G)=$ $C_{O_{p^{\prime}}(G)}(P) \times\left[O_{p^{\prime}}(G), P\right]$ for any Sylow $p$-subgroup $P$ of $G$. Thus, $\left[O_{p^{\prime}}(G), P\right]=1$ and, as the $p$-complements of $G$ are abelian, we deduce that necessarily $O_{p^{\prime}}(G) \subseteq Z(G)$, 
so $F(G)=O_{p}(G) \times Z(G)_{p^{\prime}}$. Now, if $y$ is a $p^{\prime}$-element of primary order of $C_{G}(z)$ which is noncentral in $G$, then $C_{G}(z)=C_{G}(y)$. By applying Lemma 2.4, we obtain $y \in C_{G}(O p(G))=C_{G}(F(G)) \subseteq F(G)$, so $y \in Z(G)$. Consequently, the $p$-complements of $G$ lie in $Z(G)$, which is a contradiction. The theorem is proved.

\section{References}

[1] E. Alemany, A. Beltrán and M. J. Felipe, 'Itô's theorem on groups with two conjugacy class sizes revisited', Bull. Austral. Math. Soc. 85 (2012), 476-481.

[2] R. Baer, 'Group elements of prime power index', Trans. Amer. Math. Soc. 75 (1953), 20-47.

[3] A. Beltrán and M. J. Felipe, 'Some class size conditions implying solvability of finite groups', $J$. Group Theory 9 (2006), 787-797.

[4] A. Beltrán and M. J. Felipe, 'Finite groups with four conjugacy class sizes', Comm. Algebra 39 (2011), 1260-1272.

[5] I. M. Isaacs, 'Subgroups generated by small classes in finite groups', Proc. Amer. Math. Soc. 136 (2008), 2299-2301.

[6] N. Itô, 'On finite groups with given conjugate types.I', Nagoya Math. J. 6 (1953), 17-28.

[7] N. Itô, 'On finite groups with given conjugate types. II', Osaka J. Math. 7 (1970), 231-251.

[8] N. Itô, 'On finite groups with given conjugate types. III', Math. Z. 117 (1970), 267-271.

[9] Q. Kong, 'Conjugacy class sizes and solvability of finite groups', Monatsh. Math. 168 (2012), 267-271.

[10] Q. Kong, 'Finite groups with four class sizes of elements of order divisible by at most three distinct primes', J. Group Theory 15 (2012), 661-667.

[11] Q. Kong and X. Guo, 'On an extension of a theorem on conjugacy class sizes', Israel J. Math. 179 (2010), 279-284.

QINGJUN KONG, Department of Mathematics, Tianjin Polytechnic University, Tianjin 300387, China

e-mail: kqj2929@163.com

QINGFENG LIU, Department of Basic Sciences, Shandong Water Polytechnic, Rizhao 276826, China 